
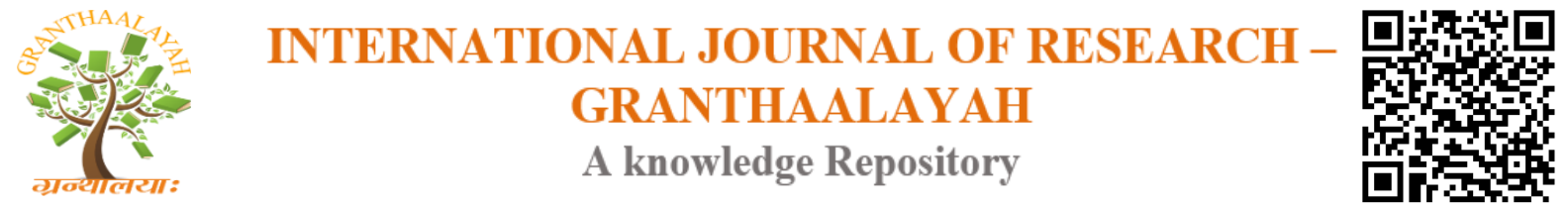

Science

\title{
DRAG \& AVERSION PARTICLE SWARM OPTIMIZATION ALGORITHM FOR REDUCTION OF REAL POWER LOSS
}

\author{
Dr.K.Lenin *1 \\ ${ }^{* 1}$ Professor, Department of EEE, Prasad V.Potluri Siddhartha Institute of Technology, Kanuru, \\ Vijayawada, Andhra Pradesh -520007, India
}

\begin{abstract}
This paper projects Drag \& Aversion Particle Swarm Optimization (DAPSO) algorithm is applied to solve optimal reactive power problem. In DAPSO the idea of decreasing and increasing diversity operators used to control the population into the basic Particle Swarm Optimization (PSO) model. The modified model uses a diversity measure to have the algorithm alternate between exploring and exploiting behavior. The results show that both Drag \& Aversion Particle Swarm Optimization (DAPSO) prevents premature convergence to enhanced level but still keeps a rapid convergence. Proposed Drag \& Aversion Particle Swarm Optimization (DAPSO) has been tested in standard IEEE 118 \& practical 191 bus test systems. Real power loss has been considerably reduced and voltage profiles are within the limits.
\end{abstract}

Keywords: Reactive Power Optimization; Real Power Loss; Particle Swarm Optimization; Drag; Aversion.

Cite This Article: Dr.K.Lenin. (2017). "DRAG \& AVERSION PARTICLE SWARM OPTIMIZATION ALGORITHM FOR REDUCTION OF REAL POWER LOSS.” International Journal of Research - Granthaalayah, 5(11), 168-176.

\section{Introduction}

The main objective of optimal reactive power problem is to minimize the real power loss and bus voltage deviation. Various numerical methods like the gradient method [1-2], Newton method [3] and linear programming [4-7] have been adopted to solve the optimal reactive power dispatch problem. Both the gradient and Newton methods have the complexity in managing inequality constraints. If linear programming is applied then the input- output function has to be uttered as a set of linear functions which mostly lead to loss of accuracy. The problem of voltage stability and collapse play a major role in power system planning and operation [8]. Evolutionary algorithms such as genetic algorithm have been already proposed to solve the reactive power flow problem [9-11]. Evolutionary algorithm is a heuristic approach used for minimization problems by utilizing nonlinear and non-differentiable continuous space functions. In [12], Hybrid differential evolution algorithm is proposed to improve the voltage stability index. In [13] Biogeography Based algorithm is projected to solve the reactive power dispatch problem. In 
[14], a fuzzy based method is used to solve the optimal reactive power scheduling method. In [15], an improved evolutionary programming is used to solve the optimal reactive power dispatch problem. In [16], the optimal reactive power flow problem is solved by integrating a genetic algorithm with a nonlinear interior point method. In [17], a pattern algorithm is used to solve ac-dc optimal reactive power flow model with the generator capability limits. In [18], F. Capitanescu proposes a two-step approach to evaluate Reactive power reserves with respect to operating constraints and voltage stability. In [19], a programming based approach is used to solve the optimal reactive power dispatch problem. In [20], A.Kargarian et al present a probabilistic algorithm for optimal reactive power provision in hybrid electricity markets with uncertain loads. This paper projects Drag \& Aversion Particle Swarm Optimization (DAPSO) algorithm is applied to solve optimal reactive power problem. Newly R. Ursem has suggested a model called the Diversity-Guided Evolutionary Algorithm (DGEA) [21]. He redefines the traditional mutation operator, the Gaussian mutation, to be a directed mutation instead. The important issue is that this directed mutation, in general, increases the diversity, whereas normal Gaussian mutation is not likely to do this, because it simply adds Arbitrary noise from some distribution with a mean of zero, normally $N\left(0 ; \sigma^{2}\right)$. Consequently, the DGEA applies diversitydecreasing operators (selection, recombination) and diversity-increasing operators (mutation) to alternate between two modes based upon a distance-to-average-point measure. The performance of the DGEA clearly shows its potential in multi-modal optimization. As [21] rightfully pinpoints, the diversity measure are traditionally used to analyze the evolutionary algorithms rather than guide them. We are great believers of adaptive controlling; that measuring and using different properties of the swarm/population while running, adds significant potential to the algorithm. We have therefore adopted the idea from Ursem with the decreasing and increasing diversity operators used to control the population into the basic PSO model. We find, it is a natural modification of the PSO [22-15], and the idea behind it is surprisingly simple. The modified model uses a diversity measure to have the algorithm alternate between exploring and exploiting behavior. We introduce two phases' Drag and Aversion. By measuring the diversity we let the swarm alternate between these phases. As long as the diversity is above a certain threshold $d_{\text {low }}$ the particles attract each other. When the diversity declines below $d_{\text {low }}$ the particles simply switch and start to repel each other until the threshold $d_{\text {high }}$ is met. With this simple scheme we obtain our modified model, which we have chosen to call the ARPSO model - the attractive and repulsive PSO. Proposed Drag \& Aversion Particle Swarm Optimization (DAPSO) has been tested in standard IEEE 118 \& practical 191 bus test systems. Real power loss has been considerably reduced and voltage profiles are within the limits.

\section{Problem formulation}

The objective of the reactive power optimization problem is to minimize the active power loss in the transmission Network as well as to improve the voltage profile of the system. Adjusting reactive power controllers like Generator bus voltages, reactive Power of VAR sources and transformer taps performs reactive Power scheduling.

$\operatorname{Min} \mathrm{P}_{\mathrm{L}}=\sum_{i=1}^{N B} P_{i}(X, Y, \delta)$

Subject to

a) The control vector constraints 


$$
X_{\text {min }} \leq X \leq X_{\max }
$$

b) The dependent vector constraints

$$
\mathrm{Y}_{\min } \leq \mathrm{Y} \leq \mathrm{Y}_{\max }
$$

c) The power flow constraint

$$
\mathrm{F}(\mathrm{X}, \mathrm{Y}, \delta)=0
$$

Where

$\mathrm{X}=\left[\mathrm{V}_{\mathrm{G}}, \mathrm{T}, \mathrm{Q}_{\mathrm{C}}\right]$

$$
\mathrm{Y}=\left[\mathrm{Qg}, \mathrm{V}_{\mathrm{L}}, \mathrm{I}\right]
$$

$\begin{array}{lll}\mathrm{NB} & - & \text { Number of buses in the system. } \\ \delta & - & \text { Vector of bus phase angles } \\ \mathrm{P}^{\mathrm{i}} & - & \text { Real Power injection into the } \mathrm{i}^{\text {th }} \text { bus } \\ \mathrm{V}_{\mathrm{G}} & - & \text { Vector of Generator Voltage Magnitudes } \\ \mathrm{T} & - & \text { Vector of Tap settings of on load Transformer Tap changer. } \\ \mathrm{Q}_{\mathrm{C}} & - & \text { Vector of reactive Power of switchable VAR sources. } \\ \mathrm{V}_{\mathrm{L}} & - & \text { Vector of load bus Voltage magnitude. } \\ \mathrm{I} & - & \text { Vector of current in the lines. } \\ \mathrm{P}_{\mathrm{L}} & - & \text { Vector of current in the lines. }\end{array}$

\section{Basic Particle Swarm Optimization}

The basic Particle Swarm Optimization (PSO) model consists of a swarm of particles moving in an n-dimensional, real valued search space of possible problem solutions. For the search space, in general, a certain quality measure, the fitness, is defined making it possible for particles to compare different problem solutions. Every particle has a position vector $\mathrm{x}$ and a velocity vector v. Moreover, each particle contains a small memory storing its own best position seen so far $\mathrm{p}$ and a global best position $g$ obtained through communication with its fellow neighbor particles. This information flow is obtained by defining a neighborhood topology on the swarm telling particles about immediate neighbors.

The intuition behind the PSO model is that by letting information about good solutions spread out through the swarm, the particles will tend to move to good areas in the search space. At each time step $t$ the velocity is updated and the particle is moved to a new position. This new position is simply calculated as the sum of the previous position and the new velocity:

$$
\vec{x}(t+1)=\vec{x}(t)+\vec{v}(t+1)
$$

The update of the velocity from the previous velocity to the new velocity is, as implemented in this paper, determined by: 
$\vec{v}(t+1)=\omega \cdot \vec{v}(t)+\phi_{1}(\vec{p}(t))+\phi_{2}(\vec{g}(t)-\vec{x}(t))$

Where $\phi_{1}$ and $\phi_{2}$ are real numbers chosen uniformly and at Arbitrary in a given interval, usually $[0,2]$. These values determine the significance of $\vec{p}(t)$ and $\vec{g}(t)$ respectively. The parameter $\mathrm{w}$ is the inertia weight and controls the magnitude of the old velocity $\vec{v}(t)$ in the calculation of the new velocity $\vec{v}(t+1)^{2}$

\section{Drag \& Aversion Particle Swarm Optimization (DAPSO)}

We define the Drag phase merely as the basic PSO algorithm. The particles will then attract each other, since in general they attract each other in the basic PSO algorithm because of the information flow of good solutions between particles. We define the second phase aversion, by "inverting" the velocity-update formula of the particles:

$\vec{v}(t+1)=\omega \cdot \vec{v}(t)-\phi_{1}(\vec{p}(t)-\vec{x}(t))-\phi_{2}(\vec{g}(t)-\vec{x}(t))$.

In the Aversion phase the individual particle is no longer attracted to, but instead repelled by the best known particle position vector $\mathrm{g}(\mathrm{t})$ and its own previous best position vector $\mathrm{p}(\mathrm{t})$.

In the Drag phase the swarm is contracting, and consequently the diversity decreases. When the diversity drops below a lower bound, $\mathrm{d}_{\text {low }}$, we switch to the Aversion phase, in which the swarm expands due to the above inverted update-velocity formula (9). Finally, when a diversity of $d_{\text {high }}$ is reached, we switch back to the Drag phase. The result of this is an algorithm that alternates between phases of exploiting and exploring - Drag and aversion - low diversity and high diversity. The Drag \& Aversion Particle Swarm Optimization (DAPSO), algorithm is shown in Fig. 1 and 2.

Initialization ( );

While not done do

Put Direction ( ); / /new!

Update Velocity ( );

New-fangled Position ( );

Allocate Fitness ( );

Compute Diversity ( ); // new!

Figure 1: Drag \& Aversion Particle Swarm Optimization algorithm

Function Set Direction

if (dir $>0 \& \&$ diversity $<$ dLow) dir $=-1$;

if ( $\operatorname{dir}>0 \& \&$ diversity $<$ dHigh) $\operatorname{dir}=1$;

Figure 2: Set Direction

The first of the two new functions, setDirection determines which phase the algorithm is currently in, simply by setting a sign-variable, dir, either to 1 or -1 depending on the diversity. In the second function, calculateDiversity, the diversity of the swarm (in the pseudo-code stored in the variable "diversity"), is set according to the diversity-measure: 
$\operatorname{diversity}(S)=\frac{1}{|S| .|L|} \cdot \sum_{t=1}^{|S|} \sqrt{\left.\sum_{j-1}^{N} p_{i j}-\bar{p}_{j}\right)^{2}}$

where $\mathrm{S}$ is the Swarm, $[\mathrm{S}]$ is the swarmsize, [L] is the length of longest the diagonal in the search space, $\mathrm{N}$ is the dimensionality of the problem, $\mathrm{p}_{\mathrm{ij}}$ is the $\mathrm{j}^{\text {, th }}$ value of the $\mathrm{I}^{\text {, th }}$ particle and $\mathrm{pj}$ is the $j^{\text {th }}$ value of the average point $p$. Note that this diversity measure is independent of swarmsize, the dimensionality of the problem as well as the search range in each dimension.

Finally, the velocity-update formula, eqn. (9) is changed by multiplying the sign-variable direction to the two last terms in it. This decides directly whether the particles attract or repel each other:

$\vec{v}(t+1)=\omega \cdot \vec{v}(t)+\operatorname{dir}\left(\phi_{1}(\vec{p}(t)-\vec{x}(t))+\phi_{2}(\vec{g}(t)-\vec{x}(t))\right)$.

\subsection{Drag \& Aversion Particle Swarm Optimization Algorithm for Reactive Power problem}

The proposed Reactive Power Optimization algorithm using the ARPSO can be expressed as follows:

Step 1. Initial searching points and velocities of agents are generated.

Step 2. Ploss to the searching points for each agent is calculated using the load flow calculation. If the constraints are violated, the penalty is added to the loss (evaluation value of agent).

The fitness function of each particle is calculated as:

$$
\begin{aligned}
& \mathrm{f}_{\mathrm{n}}=\mathrm{P}^{\mathrm{n}}{ }_{\mathrm{L}+\alpha} \sum_{\mathrm{j}=1}^{\mathrm{NG}} \mathrm{Q}_{\mathrm{G}, \mathrm{j}}^{\lim , \mathrm{n}}+\beta \sum_{j=1}^{\mathrm{NL}} V_{\mathrm{L}, \mathrm{j}}^{\lim , \mathrm{n}} ; \mathrm{n}=1,2 . ., \mathrm{N}_{\mathrm{n}} \\
& \alpha, \beta=\quad \text { penalty factors } \\
& \mathrm{P}_{\mathrm{G}, \mathrm{j}}^{\mathrm{n}}= \begin{cases}\mathrm{lim}, \mathrm{t} & \text { total real power losses of the } \mathrm{n}^{\text {-th }} \text { particle } \\
\mathrm{Q}_{\mathrm{G}, \min }-\mathrm{Q}_{\mathrm{G}, \mathrm{j}}^{\mathrm{n}} & \text { if } \mathrm{Q}_{\mathrm{G}, \mathrm{j}}^{\mathrm{n}}<\mathrm{Q}_{\mathrm{G}, \min } \\
\mathrm{Q}_{\mathrm{G}, \mathrm{j}}^{\mathrm{n}}-\mathrm{Q}_{\mathrm{G}, \max } & \text { if } \mathrm{Q}_{\mathrm{G}, \mathrm{j}}^{\mathrm{n}}>\mathrm{Q}_{\mathrm{G}, \max }\end{cases}
\end{aligned}
$$

and

$$
V_{\mathrm{L}, \mathrm{j}}^{\lim , \mathrm{n}}= \begin{cases}\left|V_{\mathrm{L}, \mathrm{j}}^{\mathrm{n}}\right|-\mathrm{V}_{\mathrm{L}, \max }, & \text { if }\left|\mathrm{V}_{\mathrm{L}, \mathrm{j}}^{\mathrm{n}}\right|>\mathrm{V}_{\mathrm{L}, \max } \\ 0 & \text { otherwise }\end{cases}
$$

Step 3. Pbest is set to each initial searching point. The initial best evaluated value (loss with penalty) among pbests is set to gbest.

Step 4. New velocities are calculated using eqn. (7).

Step 5. Update the velocity from previous velocity to the new velocity using eqn. (8).

Step 6. To new function applied.

i. setdirection

ii. Calculate Diversity to control swarm. 
Step 7.Ploss to the new searching points and the evaluation values are calculated.

Step 8. If the evaluation value of each agent is better than the previous pbest, the value is set to pbest. If the best pbest is better than gbest, the value is set to gbest. All of gbests are stored as candidates for the final control strategy.

Step 9. If the iteration number reaches the maximum iteration number, then stop. Otherwise, go to Step 4. If the voltage and power flow constraints are violated, the absolute violated value from the maximum and minimum boundaries is largely weighted and added to the objective function (1) as a penalty term. The maximum iteration number should be determined by pre-simulation. As mentioned below, PSO requires less than 100 iterations even for large-scale problems.

\section{Simulation Results}

At first Drag \& Aversion Particle Swarm Optimization (DAPSO) algorithm, has been tested in standard IEEE 118-bus test system [26]. The system has 54 generator buses, 64 load buses, 186 branches and 9 of them are with the tap setting transformers. The limits of voltage on generator buses are $0.95-1.1$ per-unit., and on load buses are $0.95-1.05$ per-unit. The limit of transformer rate is $0.9-1.1$, with the changes step of 0.025 . The limitations of reactive power source are listed in Table 1, with the change in step of 0.01 .

Table 1: Limitation of reactive power sources

\begin{tabular}{|l|l|l|l|l|l|l|l|}
\hline BUS & 5 & 34 & 37 & 44 & 45 & 46 & 48 \\
\hline QCMAX & 0 & 14 & 0 & 10 & 10 & 10 & 15 \\
\hline QCMIN & -40 & 0 & -25 & 0 & 0 & 0 & 0 \\
\hline BUS & 74 & 79 & 82 & 83 & 105 & 107 & 110 \\
\hline QCMAX & 12 & 20 & 20 & 10 & 20 & 6 & 6 \\
\hline QCMIN & 0 & 0 & 0 & 0 & 0 & 0 & 0 \\
\hline
\end{tabular}

The statistical comparison results of 50 trial runs have been list in Table 2 and the results clearly show the better performance of proposed Drag \& Aversion Particle Swarm Optimization (DAPSO) approach.

Table 2: Comparison results

\begin{tabular}{|l|l|l|l|l|}
\hline Active power loss (p.u) & $\begin{array}{l}\text { BBO } \\
{[\mathbf{2 7}]}\end{array}$ & $\begin{array}{l}\text { ILSBBO/ } \\
\text { strategy1 } \\
{[\mathbf{2 7}]}\end{array}$ & $\begin{array}{l}\text { ILSBBO/ } \\
\text { strategy1 } \\
{[\mathbf{2 7}]}\end{array}$ & $\begin{array}{l}\text { Proposed } \\
\text { DAPSO }\end{array}$ \\
\hline Min & 128.77 & 126.98 & 124.78 & 116.98 \\
\hline Max & 132.64 & 137.34 & 132.39 & 121.84 \\
\hline Average & 130.21 & 130.37 & 129.22 & 119.36 \\
\hline
\end{tabular}

Then the Drag \& Aversion Particle Swarm Optimization (DAPSO) algorithm has been tested in practical 191 test system and the following results have been obtained. In Practical 191 test bus system - Number of Generators $=20$, Number of lines $=200$, Number of buses $=191$ Number of transmission lines $=55$. Table 3 shows the optimal control values of practical 191 test system obtained by DAPSO method. And table 4 shows the results about the value of the real power loss by obtained by Drag \& Aversion Particle Swarm Optimization (DAPSO). 
Table 3. Optimal Control values of Practical 191 utility (Indian) system by DAPSO method

\begin{tabular}{l|l|l|l|l}
\hline VG1 & 1.100 & & VG 11 & 0.900 \\
\hline VG 2 & 0.720 & VG 12 & 1.000 \\
\hline VG 3 & 1.010 & VG 13 & 1.000 \\
\hline VG 4 & 1.010 & VG 14 & 0.900 \\
\hline VG 5 & 1.100 & VG 15 & 1.000 \\
\hline VG 6 & 1.100 & VG 16 & 1.000 \\
\hline VG 7 & 1.100 & VG 17 & 0.900 \\
\hline VG 8 & 1.010 & VG 18 & 1.000 \\
\hline VG 9 & 1.100 & VG 19 & 1.100 \\
\hline VG 10 & 1.010 & VG 20 & 1.100 \\
\hline
\end{tabular}

\begin{tabular}{|c|c|c|c|c|c|}
\hline $\mathrm{T} 1$ & 1.000 & T21 & 0.900 & $\mathrm{~T} 41$ & 0.900 \\
\hline $\mathrm{T} 2$ & 1.000 & $\mathrm{~T} 22$ & 0.900 & T42 & 0.900 \\
\hline $\mathrm{T} 3$ & 1.000 & $\mathrm{~T} 23$ & 0.900 & T43 & 0.910 \\
\hline $\mathrm{T} 4$ & 1.100 & T24 & 0.900 & T44 & 0.910 \\
\hline T5 & 1.000 & $\mathrm{~T} 25$ & 0.900 & $\mathrm{~T} 45$ & 0.910 \\
\hline T6 & 1.000 & T26 & 1.000 & T46 & 0.900 \\
\hline $\mathrm{T} 7$ & 1.000 & $\mathrm{~T} 27$ & 0.900 & T47 & 0.910 \\
\hline T8 & 1.010 & T28 & 0.900 & T48 & 1.000 \\
\hline T9 & 1.000 & T29 & 1.010 & T49 & 0.900 \\
\hline $\mathrm{T} 10$ & 1.000 & $\mathrm{~T} 30$ & 0.900 & T50 & 0.900 \\
\hline T11 & 0.900 & T31 & 0.900 & T51 & 0.900 \\
\hline T12 & 1.000 & T32 & 0.900 & T52 & 0.900 \\
\hline T13 & 1.010 & T33 & 1.010 & T53 & 1.000 \\
\hline T14 & 1.010 & T34 & 0.900 & T54 & 0.900 \\
\hline T15 & 1.010 & T35 & 0.900 & T55 & 0.900 \\
\hline T19 & 1.020 & T39 & 0.900 & & \\
\hline $\mathrm{T} 20$ & 1.010 & $\mathrm{~T} 40$ & 0.900 & & \\
\hline
\end{tabular}

Table 4: Optimum real power loss values obtained for practical 191 utility (Indian) system by DAPSO method.

\begin{tabular}{l|l}
\hline Real power Loss (MW) & DAPSO \\
\hline Min & 145.102 \\
\hline Max & 149.114 \\
\hline Average & 147.786 \\
\hline
\end{tabular}

\section{Conclusion}

In this paper Drag \& Aversion Particle Swarm Optimization (DAPSO) algorithm has been successfully solved the Reactive power optimization problem. In DAPSO the idea of decreasing and increasing diversity operators used to control the population into the basic Particle Swarm Optimization (PSO) model. The modified model uses a diversity measure to have the algorithm 
alternate between exploring and exploiting behavior. The results show that both Drag \& Aversion Particle Swarm Optimization (DAPSO) prevents premature convergence to extraordinary level but still keeps a quick convergence. Proposed Drag \& Aversion Particle Swarm Optimization (DAPSO) algorithm has been tested in standard IEEE 118 \& practical 191 bus test systems. Real power loss has been considerably reduced and voltage profiles are within the limits.

\section{References}

[1] O.Alsac, B. Scott, "Optimal load flow with steady state security", IEEE Transaction. PAS -1973, pp. 745-751.

[2] Lee K Y ,Paru Y M , Oritz J L -A united approach to optimal real and reactive power dispatch , IEEE Transactions on power Apparatus and systems 1985: PAS-104 : 1147-1153

[3] A.Monticelli , M .V.F Pereira ,and S. Granville, "Security constrained optimal power flow with post contingency corrective rescheduling", IEEE Transactions on Power Systems :PWRS-2, No. 1, pp.175-182., 1987.

[4] Deeb N, Shahidehpur S.M, Linear reactive power optimization in a large power network using the decomposition approach. IEEE Transactions on power system 1990: 5(2) : 428-435

[5] E. Hobson ,' Network cons rained reactive power control using linear programming, ' IEEE Transactions on power systems PAS -99 (4),pp 868=877, 1980

[6] K.Y Lee, Y.M Park, and J.L Oritz, "Fuel -cost optimization for both real and reactive power dispatches", IEE Proc; 131C,(3), pp.85-93.

[7] M.K. Mangoli, and K.Y. Lee, "Optimal real and reactive power control using linear programming”, Electr. Power Syst. Res, Vol.26, pp.1-10, 1993.

[8] C.A. Canizares, A.C.Z.de Souza and V.H. Quintana, "Comparison of performance indices for detection of proximity to voltage collapse,' vol. 11. no.3, pp.1441-1450, Aug 1996.

[9] K.Anburaja, "Optimal power flow using refined genetic algorithm", Electr. Power Compon. Syst, Vol. 30, 1055-1063, 2002.

[10] D. Devaraj, and B. Yeganarayana, "Genetic algorithm based optimal power flow for security enhancement", IEE proc-Generation. Transmission and. Distribution; 152, 6 November 2005.

[11] A.Berizzi, C. Bovo, M. Merlo, and M. Delfanti, "A ga approach to compare or pf objective functions including secondary voltage regulation," Electric Power Systems Research, vol. 84, no. 1, pp. $187-194,2012$.

[12] C.-F. Yang, G. G. Lai, C.-H. Lee, C.-T. Su, and G. W. Chang, "Optimal setting of reactive compensation devices with an improved voltage stability index for voltage stability enhancement," International Journal of Electrical Power and Energy Systems, vol. 37, no. 1, pp. $50-57,2012$.

[13] P. Roy, S. Ghoshal, and S. Thakur, "Optimal var control for improvements in voltage profiles and for real power loss minimization using biogeography based optimization," International Journal of Electrical Power and Energy Systems, vol. 43, no. 1, pp. 830 - 838, 2012.

[14] B. Venkatesh, G. Sadasivam, and M. Khan, "A new optimal reactive power scheduling method for loss minimization and voltage stability margin maximization using successive multi-objective fuzzy lp technique," IEEE Transactions on Power Systems, vol. 15, no. 2, pp. 844 -851, may 2000.

[15] W. Yan, S. Lu, and D. Yu, "A novel optimal reactive power dispatch method based on an improved hybrid evolutionary programming technique," IEEE Transactions on Power Systems, vol. 19, no. 2, pp. $913-918$, may 2004.

[16] W. Yan, F. Liu, C. Chung, and K. Wong, "A hybrid genetic algorithm interior point method for optimal reactive power flow," IEEE Transactions on Power Systems, vol. 21, no. 3, pp. 1163 1169, aug. 2006. 
[17] J. Yu, W. Yan, W. Li, C. Chung, and K. Wong, "An unfixed piece wise optimal reactive powerflow model and its algorithm for ac-dc systems," IEEE Transactions on Power Systems, vol. 23, no. 1 , pp. $170-176$, feb. 2008.

[18] F. Capitanescu, "Assessing reactive power reserves with respect to operating constraints and voltage stability," IEEE Transactions on Power Systems, vol. 26, no. 4, pp. 2224-2234, nov. 2011.

[19] Z. Hu, X. Wang, and G. Taylor, "Stochastic optimal reactive power dispatch: Formulation and solution method," International Journal of Electrical Power and Energy Systems, vol. 32, no. 6, pp. $615-621,2010$.

[20] A.Kargarian, M. Raoofat, and M. Mohammadi, "Probabilistic reactive power procurement in hybrid electricity markets with uncertain loads," Electric Power Systems Research, vol. 82, no. 1, pp. $68-80,2012$.

[21] R.K.Ursem, 'Diversity guided evolutionary algorithms', in submission for the problem solving from nature conference, (PPSN 7)

[22] Y.Fukuyama, 'A Particle Swarm Optimization for Reactive Power and voltage control in Electric Power System’, IEEE Trans. on Power Systems, pp. 87 - 93, 2001.

[23] Jakob S. Vesterstrom and Jacques Riget, "A Diversity-Guided Particle Swarm Optimizer the ARPSO”, EVALife Technical report no. 2002.

[24] Y. Fukuyama, et al., "Practical Distribution State Estimation Using Hybrid Particle Swarm Optimization", Proc. of IEEE Power Engineering Society Winter Meeting, Columbus, 2001.

[25] R. C. Eberhart and Y. Shi, "Comparing Inertia Weights and Constriction Factors in Particle Swarm Optimization", Proc. of CEC 2000.

[26] IEEE, "The IEEE 30-bus test system and the IEEE 118-test system", (1993), http://www.ee.washington.edu/trsearch/pstca/.

[27] Jiangtao Cao, Fuli Wang and Ping Li, "An Improved Biogeography-based Optimization Algorithm for Optimal Reactive Power Flow", International Journal of Control and Automation Vol.7, No.3 (2014), pp.161-176.

\footnotetext{
*Corresponding author.

E-mail address: gklenin@ gmail.com
} 